\title{
ЭЛЕКТРОЛИТНЫЕ И ЭНДОКРИННЫЕ НАРУШЕНИЯ У ПАЦИЕНТОВ, ПЕРЕНЕСШИХ ОПЕРАТИВНЫЕ ВМЕШАТЕЛЬСТВА НА ХИАЗМАЛЬНО-СЕЛЛЯРНОЙ ОБЛАСТИ
}

\author{
Юдина А.А., Никитина И.Л.
}

\author{
ФГБУ «НМИЦ им. В. А. Алмазова» Минздрава России, Санкт-Петербург
}

Опухоли хиазмально-селлярной области (ХСО) у детей занимают до 15\% от всех опухолей головного мозга. Анатомо-физиологические особенности данной зоны способствуют высокому риску развития нарушений водно-электролитного обмена и эндокринных недостаточностей в рамках гипо- и пангипопитуитаризма как при непосредственном росте опухоли, так и при нарушении связей гипоталамо-гипофизарный оси путем повреждения ножки гипофиза в ходе оперативного вмешательства на ХСО.

ЦЕЛЬ: изучение периоперационных эндокринных и водно-электролитных нарушений у пациентов, получивших хирургическое лечение опухолей ХСО.

МАТЕРИАЛЫ И МЕТОДЫ: для анализа бЫли отобраны случаи 10-ти пациентов (9 мальчиков и 1 девочки) в возрасте 10,3 лет \pm 7,6 лет, с развившимися водно-электролитными и эндокринными нарушениями после перенесенного хирургического лечения объемного образования ХСО. В раннем послеоперационном периоде пациенты наблюдались в отделении интенсивной терапии, где проводилась оценка водного и электролитного обменов, а также гормонального профиля с последующим динамическим наблюдением на базе детского лечебно-реабилитационного комплекса НМИЦ им. В.А. Алмазова.

PЕЗУЛЬТАТЫ: до операции изменения имелись в 50\% случаев. По результатам гистологического ответа $60 \%$ опухолей - представлены адамантиноподобной краниофарингиомой. В раннем послеоперационном периоде клиника центрального несахарного диабета (ЦНД) впервые развилась у 6 человек, начиная с 3 суток у 4 пациентов отмечены проявления синдрома неадекватной секреции антидиуретического гормона (SIADH), в 2 случаях отмечалось присоединение центрального сольтеряющего синдрома (CSW). Спустя 14 дней после хирургического лечения стойкий ЦНД имел место у 5 пациентов. К моменту выписки из хирургического стационара в заместительной гормональной терапии (ЗГТ) нуждались 8 человек. Впервые в раннем послеоперационном периоде диагностированы центральные гипокортицизм и гипотиреоз в 6 и 5 случаях соответственно. При анализе катамнеза прогрессия клиники, ранее имевшихся до операции нарушений, с потребностью в увеличении доз ЗГТ отмечалась в 3 случаях.

\section{Выводы:}

1. Рост опухоли ХСО сам по себе может привести к развитию водно-электролитных и эндокринных нарушений. В дооперационном периоде заведомо имели нарушения 5 человек;

2. В послеоперационном периоде клиническая картина водно-электролитных нарушений в данной когорте пациентов представлена ЦНД (75\%), у 3 из которых отмечен «трехфазный» вариант течения, в 2 случаях зафиксировано сочетание транзиторного течения ЦНД с CSW. Стойкий ЦНД имел место у 5 человек;

3. В 75\% случаев эндокринные нарушения центрального генеза (гипокортицизм, гипотиреоз) впервые развились в течение первых 2x недель. Имели прогрессию у 3 пациентов с потребностью в коррекции терапии;

4. Комплексный подход в ведении пациентов после удаления образований ХСО определяет максимальную эффективность коррекции нарушений в раннем послеоперационном периоде. 